\title{
COMPOSITE MODELS FOR NARROW LINE REGIONS
}

\author{
STEFANIE KOMOSSA \\ Astronom. Institut, Ruhr-Universität, D-44780 Bochum
}

The basic excitation mechanism for the emission lines of Seyfert galaxies is generally believed to be photoionization by radiation emerging from the central power source. In order to deduce the essential physical parameters appropriate to the narrow line region (NLR) on this basis and to overcome some systematic deficiencies of existing photoionization models, we drop the frequent assumption of a constant ionization parameter throughout the NLR and, in particular, allow for a range of densities at fixed radius. We predict all observed emission-lines (ranging from CIV $\lambda 1549$ in the UV- to [SIII] $\lambda 9069$ in the NIR spectral region) for the sample of Sy 2 galaxies using the code CLOUDY (Ferland 1993). Our models are characterized by (values in parentheses give the considered range for each parameter):

1) the shape and strength of the ionizing continuum (power laws with $\alpha_{u v-x}=$ $-1 \ldots-2.5$, black bodies with $T_{b b}=(1.0 \ldots 2.5) \times 10^{5} \mathrm{~K}$, combinations of both; $\log Q=54,53)$ constrained in the non-EUV part by recent observations.

2) the geometrical and physical properties of the clouds, i.e.

i) distances from the central source $\left(r=10^{20 \ldots 21.5} \mathrm{~cm}\right.$ for $\left.Q=10^{54} \mathrm{~s}^{-1}\right)$,

ii) cloud column densities $\left(N_{h}=10^{18 \ldots 24} \mathrm{~cm}^{-2}\right.$; for the employed range of densities $10^{18}$ always corresponds to matter-bounded clouds, $10^{24}$ to ionization-bounded ones),

iii) total hydrogen density $\left(n=10^{2 \ldots 6} \mathrm{~cm}^{-3}\right)$ and density distribution over the whole region and

iv) metal abundances $(0.3 \ldots 3 \times$ solar $)$.

The major results are the following :

By composing the NLR of an ensemble of clouds distributed over a range of radii and with a range of densities at each radius we improve the predictions of high ionization lines like [NeV], CIII] and CIV and, in particular, the hitherto poorly modeled NIR-lines [SIII] and [OII].

To cover the whole observed range for each emission line, various spectral energy distributions incident on the clouds are required, involving both single power laws and black body components in the EUV-part of the continuum.

Solving the 'temperature-problem' on the basis of pure photoionization modelling can only consistently be attained by reducing the metal abundances (as compared to the contribution of a high-density component, matter-bounded clouds or the influence of dust).

No parameter-combination could be found to account on the one hand for the correlations of [NI] 25200 with other lines (namely HeII) and on the other hand for its observed strength and correlations between further lines.

T. J.-L. Courvoisier and A. Blecha: Multi-Wavelength Continuum Emission of AGN, 449.

(C) 1994 IAU. Printed in the Netherlands. 